\title{
HIPERTENSION PORTAL EN PACIENTE CON ANTECEDENTE DE ONFALOCLISIS
}

\section{Portal hypertension in patients with background of onfaloclisis}

\author{
*Eliza Funes, **Manuel González Pieri.
}

\section{RESUMEN}

La hipertensión portal es una patología cuya expresión clínica más dramática es la hemorragia digestiva por várices, constituye una emergencia de difícil manejo, con una mortalidad que puede alcanzar el $25 \%$ según el contexto. Se denomina hipertensión portal a la elevación de la presión portal por encima de $10 \mathrm{~mm} \mathrm{Hg}$ ocasionada por aumento de resistencia y flujo portales. Se presenta el Caso clínico de paciente masculino de 9 años, que llego a la emergencia de "Hospital Privado" con historia de hematemesis en dos ocasiones, de moderada cantidad, posteriormente se le realizó endoscopia digestiva alta que reportó varices esofágicas grado IV sin sangrado pero con signos de mal pronóstico y ulcera gástrica Forrest II C sin datos de sangrado reciente. En el ultrasonido doppler abdominal se observaron dilataciones venosas hacia el hilio hepático tortuosas con registro de onda fasica con flujo hepatofuga. Angiografía por Resonancia Magnética (ARM) mostró cambios en la intensidad de señal en relación a cambios por transformación cavernosa portal y evidencia de esplenomegalia. Diagnosticándolo como Hipertensión Portal, durante la estadía intrahospitalaria, en el interrogatorio la madre refirió haber sido sometido a cateterismo umbilical en el periodo neonatal. Se recomienda que a todo paciente sometido a cateterismo venoso umbilical, se le realice una radiografía toracoabdominal para observar la colocación correcta del catéter umbilical y así prevenir complicaciones futuras.

\section{PALABRAS CLAVE}

Hipertensión Portal, Sistema Porta, Esplenomegalia, fibrosis, varices esofágicas.

*Médico Radiólogo, Mediscan

** Médico General, Mediscan

Dirigir correspondencia a: mjpieri9@gmail.com

Recibido: 06 de mayo 2016 Aprobado: 08 de diciembre 2016.

\section{ABSTRACT}

Portal hypertension is a pathology whose most dramatic clinical manifestation is digestive hemorrhage due to varicose veins. It is an emergency that is difficult to manage, with a mortality that can reach $25 \%$ depending on the context. Portal hypertension is called elevation of portal pressure above $10 \mathrm{~mm} \mathrm{Hg}$ caused by increased resistance and flow of portals. The present the case is a 9-year-old male patient, who came to the emergency of "Private Hospital" with a history of hematemesis on two occasions, of moderate amount, after which he underwent a high digestive endoscopy that reported grade IV esophageal varices without bleeding but With signs of poor prognosis and gastric ulcer Forrest II C without recent bleeding data. In abdominal ultrasound Doppler hepatic venous dilatations with hepatic flow record were observed. The Magnetic Resonance Angiography (MRA) showed changes in signal intensity in relation to changes by portal cavernous transformation and evidence of splenomegaly. Diagnosing it as Portal hypertension, during the hospital stay, during the interrogation, the mother reported having undergone umbilical catheterization in the neonatal period. It is recommended that every patient undergoing umbilical venous catheterization underwent a thoracoabdominal radiograph to observe the correct placement of the umbilical catheter and thus prevent future complications.

\section{KEYWORDS}

Hypertension Portal, Porta System, splenomegaly, fibrosis, esophageal varices.

\section{INTRODUCCION}

La hipertensión portal es una patología cuya expresión clínica más dramática es la hemorragia digestiva por várices, constituye una 
emergencia de difícil manejo, con una mortalidad que puede alcanzar al $25 \%$ según el contexto en que se presente. ${ }^{(1)}$

Se denomina hipertensión portal a la elevación de la presión portal por encima de $10 \mathrm{~mm}$ Hg ocasionada por aumento de resistencia y flujo portales. Los signos y síntomas son ocasionados por la descompresión de esta hipertensión a través de colaterales porto sistémicas. La complicación más severa es el sangrado por varices esofágicas que se produce cuando la presión aumenta por encima de 12 $\mathrm{mm} \mathrm{Hg}{ }^{(2)}$

La vena porta hepática se forma por la confluencia de a) vena mesentérica superior, que trae sangre del intestino delgado y el páncreas, y b) vena esplénica, que proviene del bazo y que, en la mayoría de los casos, ha recibido a la vena mesentérica inferior y venas gástricas (coronaria, gastroepiploica y vasos cortos); la vena porta trae al hígado sangre rica en nutrientes y hormonas que vuelca en un nuevo sistema capilar intraparenquimatoso, los sinusoides, cuyas características especiales le permiten al hepatocito desarrollar las funciones de intercambio y metabolismo imprescindibles para la homeostasis. Una vez transcurrido el recorrido por los sinusoides, el drenaje venoso se efectúa a través de las venas suprahepáticas hacia la vena cava inferior, de retorno al corazón. El sistema venoso portal carece de válvulas, por lo que cualquier incremento de presión será transmitido inmediatamente en sentido retrógrado. Esta característica, unida al hecho de que los sinusoides son fenestrados y no poseen membrana basal, explica las dos consecuencias clínicas más importantes de la hipertensión portal: el desarrollo de colaterales (várices) y la trasudación de líquido hacia la cavidad peritoneal (ascitis). Las causas de la hipertensión portal están relacionadas con un bloqueo en el flujo portal y el incremento de la resistencia es el factor iniciador del proceso. Este incremento puede localizarse antes de los sinusoides denominándose hipertensión portal pre sinusoidal (hipertensión pre hepática), a nivel de los sinusoides denominada sinusoidal (hipertensión intrahepatica), o después de ellos (post hepática) llamada post sinusoidal. ${ }^{(3)}$

La obstrucción pre hepática (pre sinusoidal) al flujo venoso constituye aproximadamente el $50 \%$ de los casos de hipertensión portal entre los niños.

La causa más común es la trombosis de la vena porta o sus ramas, el cuadro típico de estos pacientes es el de una esplenomegalia asintomática que evoluciona en la mayoría de casos durante varios años (aunque también pueden presentarse de forma precoz), asociada a citopenias, especialmente plaquetopenia, por el hiperesplenismo que se desarrolla secundariamente al aumento de presión en el sistema porta. Eventualmente, el paciente desarrolla hemorragia digestiva (hematemesis, melena), lo que permite detectar las várices y establecer el diagnóstico. Algunos pacientes con sangrado grave pueden desarrollar ascitis posterior al episodio hemorrágico, probablemente en relación con una caída transitoria en las cifras de albúmina sérica. ${ }^{(3)}$

La ecografía de abdomen con Doppler es el método preferido para el diagnóstico de la obstrucción de la vena porta, por ser mínimamente invasivo y altamente sensible (94 a $100 \%$ ) y específico (90 a 96\%) cuando está en manos de un operador experimentado. La permeabilidad de los vasos, la dirección y velocidad del flujo (valor máximo $<20 \mathrm{~cm} / \mathrm{s}$ y mínimo $<10-12 \mathrm{~cm} / \mathrm{s}$ ) la presencia de colaterales y el tamaño del bazo son algunos de los elementos fundamentales que se deben evaluar. $^{(3,4)}$

La etiología puede ser:

A) Cirrosis 50\%:

1. Colestasis $70 \%$ y puede deberse a atresia biliar, Síndrome de Alagille, colesta- 
sis intrahepatica familiar progresiva, Colangitis esclerosante.

2. Postnecrotica $15 \%$ puede ser autoinmune o por Hepatitis B y C.

3. Metabólica $10 \%$ debido a déficit de alfa1-antitripsina, Tirosinemia tipo 1, Fibrosis Quistica o enfermedad de Wilson.

4. Idiopáticas $5 \%$.

B) Hígado no cirrótico:

1. Extrahepática: obstrucción venosa portal $36 \%$, congénitas $25 \%$, cateterización umbilical $30 \%$, onfalitis.

2. Intrahepatica: Presinusoidal: fibrosis hepática congénita $7 \%$, esquistosomia sis, granulomas, hemangiomas. Parasinusoidal: hígado graso, hiperplasia nodular focal, hiperplasia nodular regenerativa. Postsinusoidal: enfermedad venoclusiva, trombosis de la vena hepática, Metastasis.

3. Suprahepatica $6 \%$, Sindrome de Buddchiari: agenesia

C) Fistula Arteriovenosa Hepática: Congénita o Adquirida. ${ }^{(4,5)}$

El recorrido normal del catéter umbilical, se muestra en la figura No. 1. Las posiciones anómalas de los catéteres umbilicales son frecuentes ya que su instalación no es guiada por imágenes. La mal posición del cateter umbilical venoso (CUV) se puede producir antes de llegar a la posición deseada y lo podemos encontrar localizado en el receso umbilical antes de llegar a la vena porta izquierda y al tratar de avanzar el catéter se puede devolver en la vena umbilical. Cuando el catéter llega al receso umbilical debe pasar a través de la porta izquierda hacia el conducto venoso, sin embargo, en este punto se puede desviar hacia la porta izquierda o a la porta derecha o incluso a la porta principal y de ahí puede pasar a la vena mesentérica superior y esplénica. El catéter en la porta puede producir trombosis portal.
La complicación más tardía y consecuencia de esta última es la cavernomatosis portal e hipertensión portal. También se han descrito hematomas hepáticos y neumatosis portal secundaria. ${ }^{(6-8)}$

\section{Figura No. 1: Algoritmo del recorrido normal del cateterismo umbilical.}

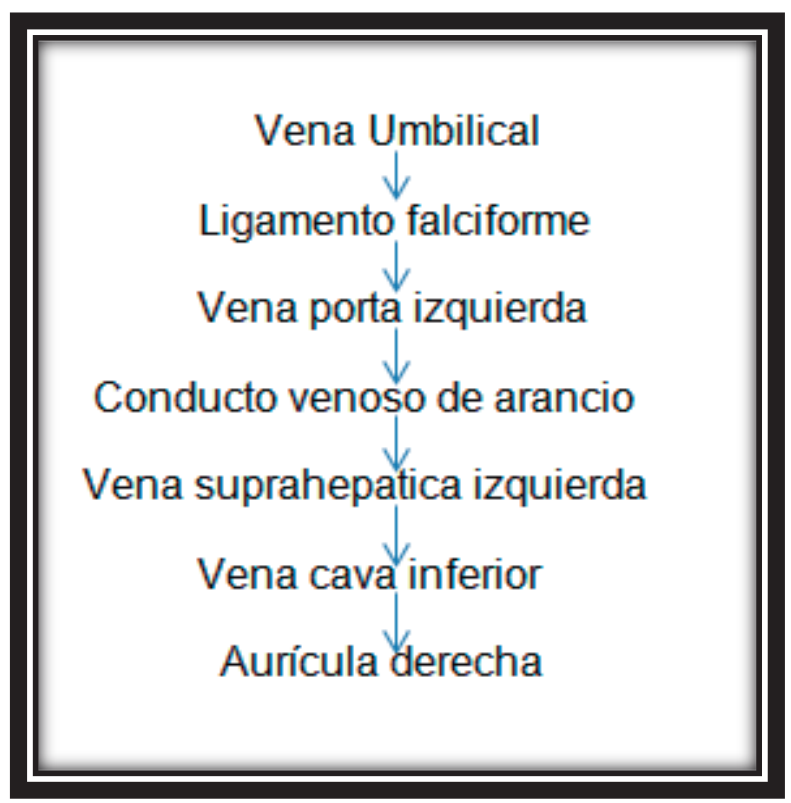

Fuente: elaboración propia

\section{CASO CLINICO}

Paciente masculino de 9 años que acude a hospital privado, con historia de hematemesis en dos ocasiones, el primer episodio de moderada cantidad con coagulo, segundo episodio con sangre rutilante acompañado de melena, proctorragia, fiebre, mialgia y artralgia. Con dolor abdominal de un día de evolución, tipo opresivo sin atenuantes ni exacerbantes. Sin antecedente de traumas ni ingesta de medicamentos. Producto de cesárea a las 30 semanas de gestación por antecedente de placenta previa, por lo que permaneció hospitalizado durante un mes, consignándose en historia clínica antecedente de cateterización umbilical en periodo neonatal. Posterior a su ingreso se realiza endoscopia digestiva alta como se observa en la figura No. 2. 


\section{Figura No. 2: Endoscopia Digestiva Alta}

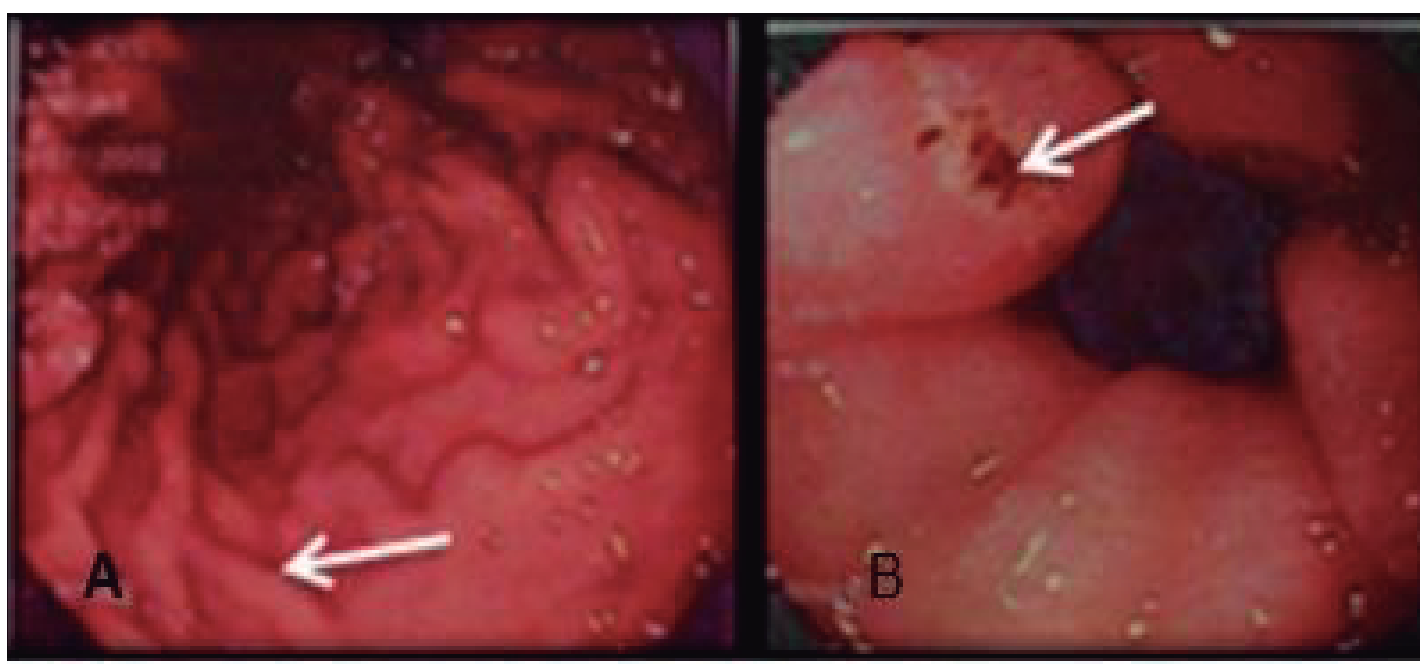

Figura No. 2. A) Varices esofágicas G IV sin sangrado, pero con signos de mal pronóstico y B) Ulcera Gástrica Forrest II C sin datos de sangrado reciente.

Además, se le realizó ultrasonido abdominal doppler color que reportó onda fasica con flujo hepatofuga, como se observa en la figura No. 3 y una Angiografía por Resonancia Magnética de abdomen como se observa en la figura 4.

\section{Figura No. 3: Ultrasonido abdominal doppler color}

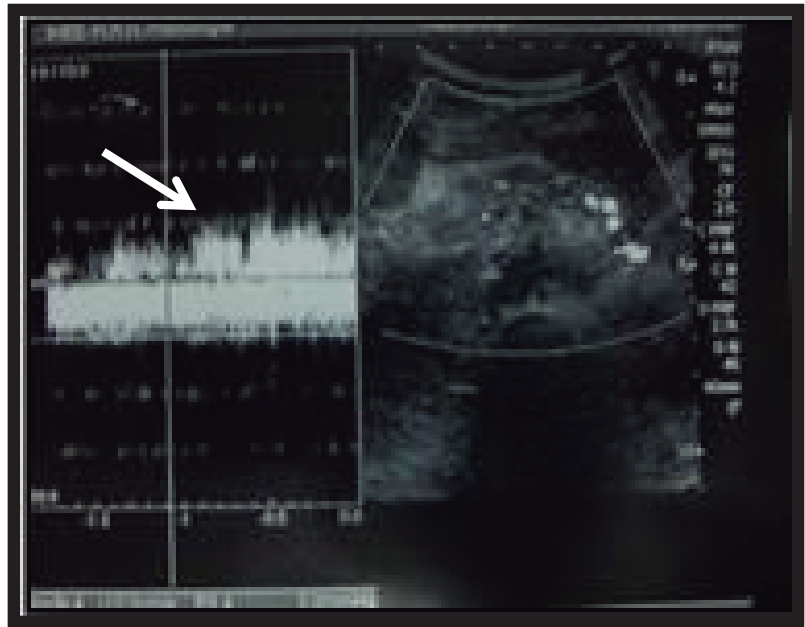

Se observan dilataciones venosas hacia el hilio hepático tortuosas con registro de onda fasica con flujo hepatofuga.
Figura No. 4: Resonancia magnética de abdomen

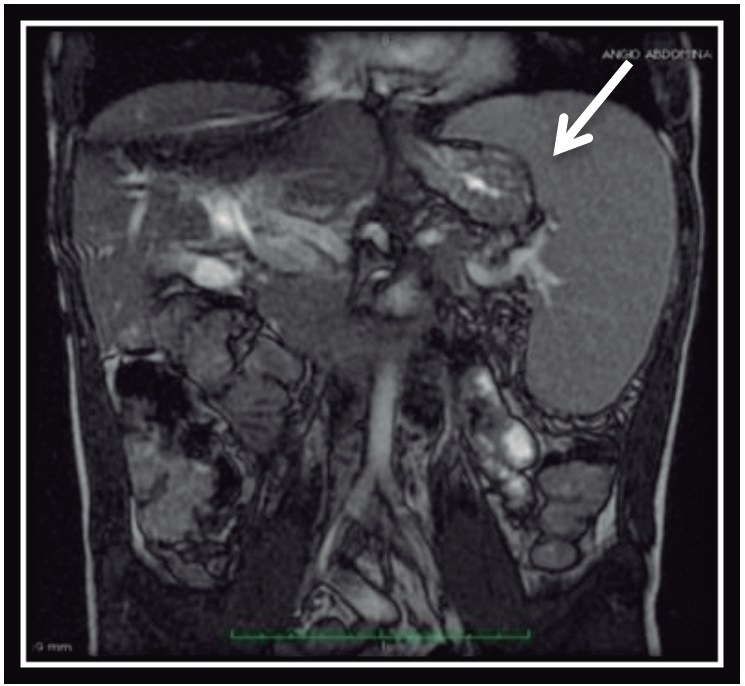

Se encontró cambios en la intensidad de señal en relación a cambios por transformación cavernosa portal y evidencia de esplenomegalia (flecha).

\section{DISCUSION}

La hipertensión portal causa complicaciones que pueden acarrear una alta mortalidad en un $30 \%$. En niños la hipertensión prehepatica o presinusoidal es la más frecuente. ${ }^{(1)}$ En el presente artículo se presenta el caso de un paciente pediátrico de 9 años con diagnóstico de hipertensión portal. La hipertensión portal 
prehepatica o presinusoidal es más frecuente en los mayores de 5 años, lo cual concuerda con lo reportado en el estudio realizado en el King's College Hospital, donde el $58 \%$ de sus pacientes fueron mayores de 5 años. ${ }^{(9)}$

El motivo de ingreso del paciente fue la hemorragia digestiva alta con 2 episodios de hematemesis previo al ingreso al hospital, al igual que en el estudio realizado por Valdivieso, en el Instituto Nacional de Salud del Niño en Lima Perú, donde la causa de ingreso hospitalario fue hematemesis en un $74 \% .^{(10)}$ La hipertensión portal es una enfermedad de signos y síntomas característicos, siendo la hematemesis, la que con mayor frecuencia aparece $74 \%$ y melena $46 \%$, demostrándose así que la hemorragia digestiva alta es en su mayoría el motivo de consulta. ${ }^{(4,8)}$

El paciente presento hiperesplenismo, siendo esta una complicación que puede evolucionar silenciosamente durante varios años, asociado a citopenias más o menos graves, especialmente plaquetopenia y muchas veces llevan a evaluaciones de la medula ósea. ${ }^{(6,9)}$

El paciente tenía el antecedente de cateterismo umbilical en el período neonatal, en este procedimiento existe manipulación del cordón umbilical que puede llevar, al cabo de un tiempo no determinado, a la aparición de manifestaciones clínicas propias de la hipertensión portal con un riesgo del $25-30 \%$ como lo dice la literatura. En el estudio de Hipertensión Portal del King College $12 \%$ de sus pacientes tuvieron cateterismo umbilical y $7 \%$ sepsis neonatal. ${ }^{(6,7,9)}$

En la endoscopia realizada al paciente, se evidencio la presencia de varices esofágicas GIII y una ulcera gástrica Forrest II C sin datos de sangrado. En un estudio realizado por Valdivieso en Perú, se realizó endoscopia digestiva alta a $2 / 3$ de los pacientes pediátricos y el $70 \%$ tuvo algún grado de varices esofágicas. ${ }^{(10)}$ El examen endoscópico es un método apropiado para evaluar el alto riesgo de sangrado así como para dar tratamiento a las varices esofágicas.

Los catéteres umbilicales son fácilmente diferenciables en la radiografía tóraco-abdominal. El CUV presenta un trayecto ascendente desde la región umbilical hacia el hígado donde se curva ligeramente hasta alcanzar la vena cava inferior o la unión de la vena cava con aurícula derecha. En la proyección lateral adquiere una forma de "s" atravesando el hígado antes de alcanzar la cava inferior. La correcta posición de la punta del CUV es en el extremo más cefálico de la vena cava inferior ( $\mathrm{VCl}$ ), en la unión de la $\mathrm{VCl}$ con la aurícula derecha (AD). En general se recomienda que el catéter finalice alejado de los principales vasos aórticos, para ello es importante conocer que: el tronco celíaco se sitúa en D12, la arteria mesentérica superior en D12-L1, la arteria renal en L1-L2 y la arteria mesentérica inferior en L3 y bifurcación aórtica en L4. ${ }^{(9)}$

En relación a los exámenes auxiliares, la ecografía y aún más la ecografía doppler es un método no invasivo que nos permite un diagnóstico precoz de esta enfermedad; así mismo permite el diagnostico de trombosis venosa portal con alta sensibilidad y especificidad, evaluando si la dilatación de la porta es mayor de $10 \mathrm{~mm}$ o si hay disminución de la velocidad de flujo de la porta (máxima $<20 \mathrm{~cm} / \mathrm{s}$ y media $<12 \mathrm{~cm} / \mathrm{s},{ }^{(9)}$ así como al paciente se le realizó el ultrasonido abdominal doppler el cual demostró dilataciones venosas hacia el hilio hepático tortuosas con registro de onda fasica y flujo hepatofuga indicativos de hipertensión portal.

Este caso pone de relieve las complicaciones hepáticas relacionadas con la posición defectuosa de Cateterismo Venoso Umbilical.

En conclusión; dado que se conocen las probables etiologías de la Hipertensión Portal en el paciente Pediátrico es importante a) implementar los Protocolos de Cateterismo Umbilical para identificar población en riesgo. 
b) una vez identificado el tipo de paciente en riesgo monitorizar su seguimiento con los exámenes clínicos y de diagnóstico pertinentes.

Se recomienda; estudio de Seguimiento a corto, mediano y largo plazo por ultrasonido doppler al paciente pediátrico que fue sometido a cateterismo umbilical pues cobran impor- tancia para determinar trombos en forma precoz o datos de hipertensión portal y así obtener diagnósticos oportunos y prevenir complicaciones como sangrado profuso por varices esofágicas, hepatoesplenomegalia, ascitis, dilataciones venosas colaterales como signos de hipertensión portal con las consecuencias posteriores de daño hepático crónico.

\section{REFERENCIAS BIBLIOGRÁFICAS}

1. Nucette A, Navarro D, Colina N, López K, Durango $R$, Arrieta A, et al. Hipertensión portal en niños: historia natural, evolución, tratamiento y pronóstico. Gen. [Internet] 2010 [Citado 20 de febrero del 2016];64(4):323-328.

Disponible en: http://www.scielo.org.ve/s cielo.php?script $=$ sci_arttext $\&$ pid $=S 00$ 16-3503201000 0400011.

2. Ibarrola JL, Núñez F, Rodríguez M, Ordoñez J. Hipertensión portal. Acta Méd Grupo Ángeles. [Internet] 2011 [Citado 22 de febrero del 2016];9(2):83-91.

Disponible en: http://www.medigraphic.com/pdfs/actmed/am-2011/am112d.pdf.

3. Costaguta A, Álvarez F. Hipertensión Portal en pediatría: aspectos fisiopatológicos y clínicos. Arch Argent Pediatr. [Internet] 2010 [ Citado 3 de marzo del 2016]; 108(3):239-242. Disponible en: https:// www.researchgate.net/profile/Alejandro Costaguta/publication/44669424_Por tal_hypertension_in_pediatrics_I_Pa thophysiology_and_clinical_aspects/ links/5462ad510cf2c0c6aec1b721.pdf.

4. Grande C. Hipertensión Portal en niños. En: Acuña Quiroz MD, Alonso Franch M, Álvarez Coca J, Arguelles Martin F, Armas Ramos H, Barri Merino a, et al. Protoco los diagnósticos terapéuticos de gastroenterología, hepatología e hipertensión pediátrica. [Internet] Madrid: Hospital Infantil Universitario La Paz. 2010. [Citado el 3 de marzo 2016]. Disponible en: https://www.aeped.es/sites/default/files/ documentos/ht_portal.pdf

5. Catalina-Rodríguez MV, Rincón-Rodríguez D, Ripoll Noiseux C, Bañares-Cañizares R. Hipertensión Portal. Medicine [internet] 2012 [ Citado 7 de marzo del 2016];11(11):634-643. Disponible en: http:// studylib.es/doc/4805524/hiperten si\%C3\% B3n-portal---elsevier-institucio nes.

6. Andrade M, García W, García C. Hipertensión portal en niños: análisis de 20 años de trabajo. Rev Cubana Pediatr [Internet] 2010 [Citado 14 de abril del 2016]; 82(1). Disponible en: http://scielo. sld.cu/scielo.php?script=sci_arttext $\&$ pid= S0034-75312010000100004.

7. Schlesinger AE, Braverman RM, DiPietro MA. Neonates and umbilical venous catheters: normal appearance, anoma lous positions, complications, and potential aid to diagnosis. AJR [Internet] 2003[ Citado el 14 de abril del 2016]; 180(4): 1147-53.

Disponible en: http:// www. ajronline.org/ doi/full/10.2214/ajr.180.4. 1801147.

8. Barrington KJ. Umbilical artery catheters in the newborn: effects of position of the 
catheter tip. Cochrane Database Syst Rev. [Internet] 2000 [ Citado 3 de mayo del 2016]; (2): 1-21. Disponible en: http:// onlinelibrary.wiley.com/doi/10.1002/ 14651858.CD000505/epdf.

9. Abd El-hamid N, Taylor RM, Marinello D, Mufti GJ, Patel R, Mieli-Vergani G, et al. Aetiology and management of extrahepatic portal vein obstruction in children: king's college hospital experience. J Pediatr Gastroenterol Nutr. [Internet] 2008 [Citado 30 de octubre del 2016]; 47(5):630-4. Disponible en: https://www. ncbi.nlm.nih.
gov/pubmed/?term=Aetiology+and $+\mathrm{Ma}$ nagement+of+Extrahepatic. +Portal+ Vein+Obstruction+in+Children \%3A+King $\% 27 \mathrm{~s}+$ College+Hospital+Ex perience.

10. Valdivieso FP. Características clínicas y epidemiológicas de la hipertensión portal Presinusoidal en niños hospitalizados en el INSN. 2005-2010 [Internet] 2008 [Citado 30 de octubre del 2016]; 47(5): 630-634. Disponible en: http:// www. insn. gob.pe/investigaciones/sites/default/fi les/Informe\%20Final\%20PE-39-2010.pdf. 